\title{
Die Eiserne Lunge
}

\author{
The Iron Lung
}

\section{Bibliografie}

DOI http://dx.doi.org/

10.1055/s-0033-1344493

Pneumologie 2013; 67: 522-524

(c) Georg Thieme Verlag KC

Stuttgart · New York

ISSN 0934-8387

\section{Korrespondenzadresse}

Dr. med. Robert Kropp

Liegnitzer Straße 5

36100 Petersberg

dr.robert.kropp@gmx.de
„Sie rettete Leben, indem sie Kranke verschluckte! " ${ }^{1}$ Das erste Gerät, mit dem eine künstliche, längerfristige maschinelle Beatmung des Menschen möglich wurde, war die Eiserne Lunge. Sie wurde in den zwanziger Jahren des letzten Jahrhunderts von Philip A. Drinker (1894-1972) entwickelt, der als Ingenieur an der Harvard Medical School in Boston, MA, USA, lehrte [1,2].

Eine Eiserne Lunge ${ }^{2}$ ist eine zwei Meter lange und bis zu 600 Kilogramm schwere Beatmungsmaschine. Sie besteht aus einer metallenen luftdichten Druck-Kammer, einem Hohlzylinder, in welchen der Patient hineingelegt wird; nur der Kopf bleibt draußen. Um den Hals herum schließt das

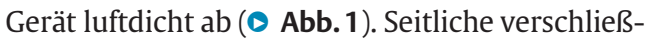
bare Öffnungen dienen der Versorgung des Kranken, zum Beispiel auch der Benutzung der Bettpfanne. Durch abwechselndes Erzeugen eines Unter- und eines Überdrucks in der Kammer mittels einer elektrischen Pumpe übernimmt der Apparat das Atmen, nämlich die Arbeit der Atemmuskulatur, das Einatmen in die und das Ausatmen aus der Lunge über die Atemwege ( $\bullet$ Abb. 2).

Die Eiserne Lunge ist leicht zu bedienen, auch von Laien. Ursprünglich war sie zur Unterstützung der Atmung bei Patienten nach Stromschlägen oder einer Gasvergiftung entwickelt worden. Noch im Versuchsstadium, noch vor ihrer offiziellen Vorstellung und der Patentanmeldung im Jahre 1929 wurde das Gerät erstmals eingesetzt, und zwar im Children's Hospital in Boston bei einem acht Jahre alten Mädchen, das an einer Poliomyelitis erkrankt und infolge der Atemlähmung schon komatös war; mit Hilfe der Eisernen Lunge konnte es rasch wiederbelebt und gerettet werden. ${ }^{3}$

\footnotetext{
${ }^{1}$ Wikipedia: de.wikipedia.org/wiki/Eiserne_Lunge

2 Volkstümlich auch Stahlsarg, Stahlkoloss, Metallmonstrum genannt, scherzhaft auch „Yellow Submarine“.

3 „Nach ein oder zwei Minuten kam das Mädchen wieder zu sich. Wenig später fragte es nach Eiscreme“, berichtete später ihr Arzt.
}

Die Poliomyelitis ${ }^{4}$, eine Infektionskrankheit, wird durch Poliomyelitis-Viren hervorgerufen und befällt vorwiegend Kinder, daher auch der deutsche Name „Kinderlähmung“. Im akuten Erkrankungsstadium kommt es zur Erkrankung der Muskelnerven und konsekutiv zu Muskellähmungen, die - häufig aufsteigend von den Füßen und Unterschenkeln - eine individuell unterschiedliche Ausdehnung haben und im Extremfall auch die Atemmuskulatur befallen und lähmen können, so dass die spontane Atmung ungenügend wird oder ganz sistiert.

Eine kurative Therapie der Poliomyelitis gibt es nicht. Deshalb ist die Prophylaxe, die Poliomyelitis-Impfung (nicht nur der Kinder), so eminent wichtig. Durch das sehr wirksame Polio 5 -Impfprogramm $^{6}$ der Welt-Gesundheits-Organisation ${ }^{7}$ konnte die Krankheit aber in weiten Teilen der Welt, in den meisten Ländern, ausgerottet werden, so auch in der Bundesrepublik Deutschland $[3,4]$.

Die Muskellähmungen, vor allem auch die Lähmung der Atemmuskulatur, sind bei der Poliomyelitis in der Regel nach dem Abklingen der akuten Infektionsphase ganz oder zumindest teilweise reversibel, also nach mehreren Tagen bis zu einigen Wochen. Für diese Zeitspanne konnte die Eiserne Lunge die Atemarbeit übernehmen und

\footnotetext{
${ }^{4}$ Früher auch Heine-Medin'sche Krankheit genannt, nach den Namen ihrer Erstbeschreiber.Vollständiger medizinischer Name: Poliomyelitis epidemica anterior acuta.

${ }^{5}$ Polio: Eher volkstümlich für Poliomyelitis

${ }^{6}$ Unterstützt, finanziert wird diese seit Jahren unternommene weltweite Kampagne zur Ausrottung der Poliomyelitis größerenteils von verschiedenen Spendern (siehe auch: Frankfurter Allgemeine Zeitung vom 26. April 2013, Seite 8, und Deutsches Ärzteblatt 2013; 110: 857 858 ), überwiegend vor allem durch das PolioPlus-Programm von Rotary International, dem Dachverband der Rotary Service Clubs, der sich seit 1979 im Kampf gegen die Kinderlähmung engagiert.

${ }^{7}$ World Health Organization $=$ WHO
} 


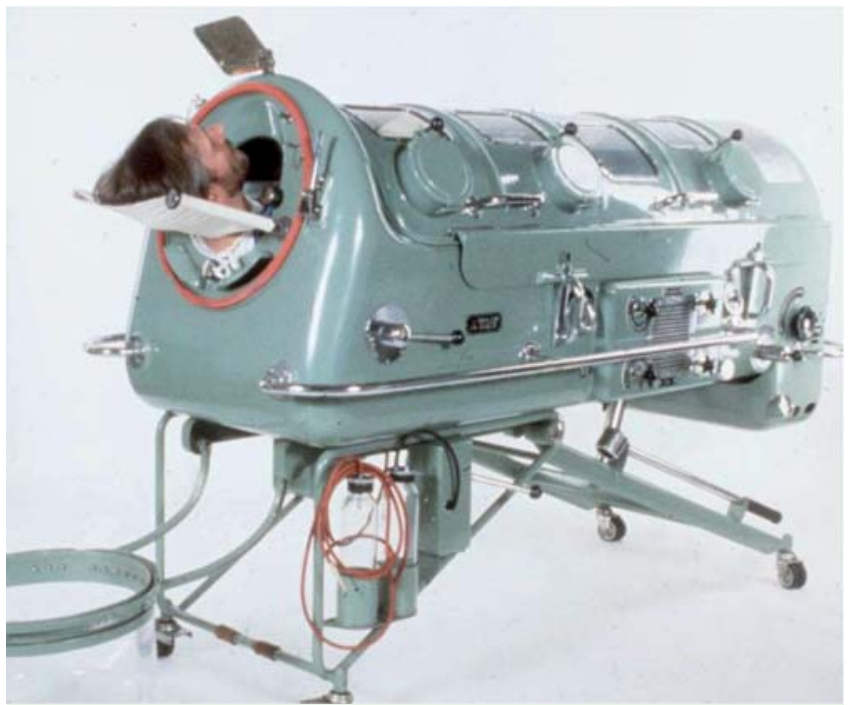

Abb. 1 Tankrespirator E 52 von Dräger aus den 50er Jahren des letzten Jahrhunderts. Durch Verschließen des durchsichtigen sog. Doms am Kopfende war bei geöffnetem Tank die Durchführung einer nicht invasiven Positivdruckbeatmung - ähnlich wie bei der modernen Helmbeatmung möglich (aus: J. Rathgeber. Grundlagen der maschinellen Beatmung. 2., vollständig überarbeitete und erweiterte Auflage. Stuttgart: Thieme; 2010).

war so im eigentlichen Wortsinn lebensrettend ( $\bullet$ Abb.3). Sie hat sich als „Rettung für die Polio-Kranken“ dem Gedächtnis eingeprägt. Natürlich war sie auch bei Atemlähmungen aus anderer Ursache hilfreich; aber die Poliomyelitis war jedenfalls schon rein zahlenmäßig die häufigste Indikation.

Der Einsatz der Eisernen Lunge konnte somit bei der Poliomyelitis häufig nach gewisser, oft kürzerer Zeit beendet werden, weil die Lähmung der Atemmuskulatur reversibel war. Doch wurde immer wieder über einzelne Patienten berichtet, bei denen die Atemmuskellähmung persistierte und die künstliche Beatmung über Jahre, ja Jahrzehnte fortgesetzt werden musste. Man kann

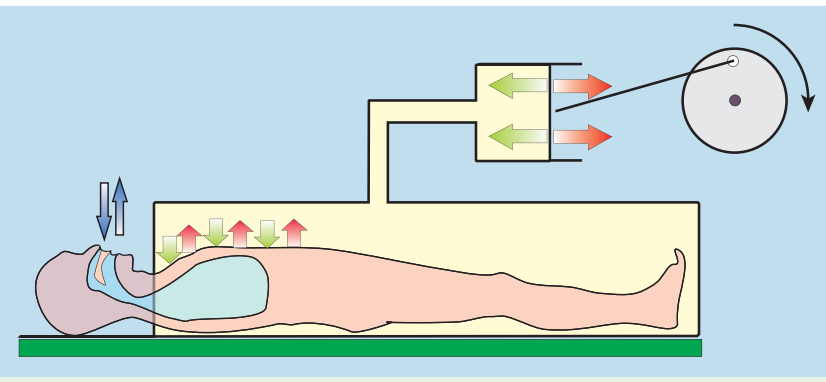

Abb.2 Das Schema veranschaulicht das Prinzip des Tankrespirators: das Gerät schließt am Hals luftdicht ab und erzeugt einen Unterdruck von -15 mbar oder mehr, wodurch die Außenluft durch Mund oder Nase des Patienten in die Lungen eingesaugt wird. Die Ausatmung wird durch Einleiten eines Überdrucks von ca. 5 mbar in die Patientenkammer erleichtert. Die Beatmungsfrequenzen liegen zwischen 10 und 20 Druckwechseln pro Minute (aus: J. Rathgeber. Grundlagen der maschinellen Beatmung. 2., vollständig überarbeitete und erweiterte Auflage. Stuttgart: Thieme; 2010).

sich die Probleme kaum vorstellen, die die Pflege, Hygiene, Ernährung dieser über einen solch langen Zeitraum ständig in der Eisernen Lunge liegenden Kranken mit sich brachten, ebenso wenig die psychische Belastung - Platzangst, das Gefühl, lebendig begraben zu sein und vieles andere.

Dies soll an drei Beispielen verdeutlicht werden. Zunächst sei hier an Martha Mason (1937-2009) aus North Carolina, USA, erinnert. Sie erkrankte im Alter von elf Jahren an einer Poliomyelitis mit nicht reversibler Atemlähmung, welche den Einsatz einer Eisernen Lunge erforderte. Sie verbrachte ein Jahr im Krankenhaus und wurde dann nach Hause entlassen, mit der Prognose, sie habe maximal noch ein Jahr zu leben. Daraus wurden über sechzig Jahre in der Eisernen Lunge; diese sollte sie bis zu ihrem Tode nicht verlassen können (zumal sie modernere und - in unseren Augen - praktischere Beatmungsmöglichkeiten, die später zur Verfügung standen, ablehnte). Man fragt sich, wie sie diese lange Zeit in einem Beatmungsgerät liegend leben konnte, mit

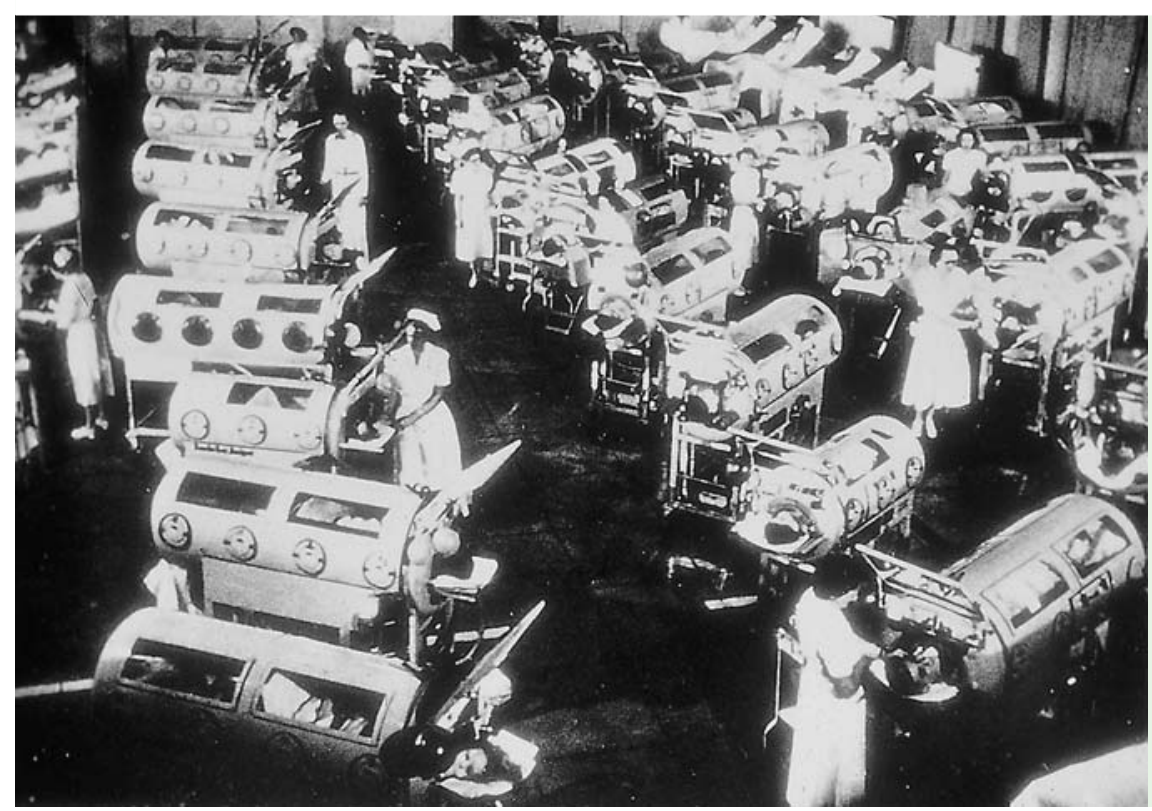

Abb.3 Beatmung mit eisernen Lungen am Universitätsklinikum (UCLA) in Los Angeles (USA) (aus: H. K. Van Aken. Intensivmedizin. 2., überarbeitete Auflage. Stuttgart: Thieme; 2006). 
dem Rhythmus, dem Lärm der Maschine, ohne „verrückt zu werden“8. Hilfe wurde ihr zuteil durch ihre Eltern, durch Pflegerinnen, die sie windelten, wendeten, wuschen. Mit Unterstützung von Hauslehrern schaffte sie den High-School-Abschluss. Danach besuchte sie eine Universität; an den Seminaren konnte sie sich von ihrer Wohnung aus mittels einer Gegensprechanlage beteiligen. Sie arbeitete später für einen Zeitungsverlag. 2003 erschien ihre Autobiographie „Breath“ [5].

Als weiteres Beispiel sei die Australierin June Middleton erwähnt, die nach einer Polio-Erkrankung im Jahre 1949 gleichfalls sechzig Jahre lang in einer Eisernen Lunge lebte, bis sie 2009 im Alter von 83 Jahren starb. Sie kam in das Guinness-Buch der Rekorde, weil sie am längsten in einer Eisernen Lunge gelebt hatte.

Und schließlich: Seit 1950, seit ihrem 4. Lebensjahr, lebte Dianne Odell aus Tennessee, USA, wegen einer Poliomyelitis-bedingten Atemlähmung in einer Eisernen Lunge. Nach 58 Jahren starb sie, weil infolge eines Sturmes die elektrische Versorgung ausfiel und gleichzeitig das Notstrom-Aggregat versagte. ${ }^{9}$

Die Eiserne Lunge ist heute nicht mehr im Gebrauch. Ihre Produktion wurde 1970 eingestellt. Ab 2004 wurde von der Herstellerfirma auch keine Wartung des Gerätes mehr angeboten, obwohl weltweit noch etwa 12 Patienten mit ihr behandelt wurden. Bessere, vorteilhaftere Beatmungsverfahren haben sie abgelöst (siehe zum Beispiel [6] und [7]). Die Eiserne Lunge ist in einigen Museen ${ }^{10}$ vorhanden und zu besichtigen.

\section{Literatur}

1 Shaw LA, Drinker PA. An apparatus for the prolonged administration of artificial respiration: I. A design for adults and children. J Clin Invest 1929; 7: 229-247

2 Shaw LA, Drinker PA. An apparatus for the prolonged administration of artificial respiration: II. A design for small children and infants with an appliance for the administration of oxygen and carbon dioxide. J Clin Invest 1929; 8: 33-46

3 Robert-Koch-Institut. Zum Welt-Poliotag 2010. Epid. Bull 2010; 42: 411-412

4 Robert-Koch-Institut. Poliomyelitis. RKI-Ratgeber für Ärzte. Epid. Bull 2000; 27/: Aktualisierte Fassung vom Februar 2011.

5 Mason M. Breath: A Lifetime in the Rhythm of an Iron Lung. Bloomsbury; 2003

6 Becker HF, Schönhofer B, Burchardi H. Kürass-Beatmung. In: Nicht-invasive Beatmung. Stuttgart: Thieme; 2005

7 Müller T, Bein T, Philipp A et al. Extrakorporale Lungenunterstützung bei schwerem Lungenversagen des Erwachsenen. Deutsches Ärzteblatt 2013; 110: 159-166
8 „60 Jahre eingesperrt“. Die Welt vom 11. 5. 2009

9 Diese drei Beispiele wurden im Internet (Google) gefunden. Sie wurden ausgewählt wegen der unglaublich langen „Bewohnung“ einer Eisernen Lunge und betreffen deshalb Polio-Patienten. Dass es sich um drei weibliche Kranke handelt, ist reiner Zufall.

10 Zum Beispiel (laut wikipedia) das Berliner Medizinhistorische Museum, das Deutsche Hygiene-Museum in Dresden, das Deutsche Museum in München, das Medizinhistorische Museum Zürich. 